\title{
Comparison of oral Lactobacillus and Streptococcus mutans between diabetic dialysis patients with non- diabetic dialysis patients and healthy people
}

\author{
Fahimeh Rezazadeh ${ }^{1}$, Abdollah Bazargani ${ }^{2}$, Jamshid Roozbeh-Shahroodi ${ }^{3}$, Ali Pooladi ${ }^{4}$, Peyman Arasteh ${ }^{5,6^{*}}$, \\ Khosro Zamani ${ }^{7}$
}

\author{
${ }^{1}$ Department of Oral and Maxillofacial Medicine, School of Dentistry, Shiraz University of Medical Sciences, Shiraz, Iran \\ ${ }^{2}$ Department of Bacteriology and Virology, Shiraz Medical School, Shiraz University of Medical Sciences, Shiraz, Iran \\ ${ }^{3}$ Shiraz Nephro-Urology Research Center, Shiraz University of Medical Sciences, Shiraz, Iran \\ ${ }^{4}$ Student Research Committee, School of Dentistry, Shiraz University of Medical Sciences, Shiraz, Iran \\ ${ }^{5}$ Non communicable Disease Research Center, Fasa University of Medical Sciences, Fasa, Iran \\ ${ }^{6}$ Student Research Committee, Shiraz University of Medical Sciences, Shiraz, Iran \\ ${ }^{7}$ Department of Bacteriology and Virology, Shiraz Medical School, Shiraz University of Medical Sciences, Shiraz, Iran
}

\section{A R T I C L E I N F O}

Article Type:

Original

\section{Article History:}

Received: 17 May 2016

Accepted: 1 July 2016

Published online: 9 July 2016

\section{Keywords:}

Lactobacillus

Streptococcus mutans

Dental caries

Diabetes mellitus

Dialysis

Renal failure

\begin{abstract}
A B S T R A C T
Introduction: Diabetes is associated with higher rates of caries, on the other hand some studies have shown that renal failure can be protective against dental caries.

Objectives: In this study we compared oral Lactobacillus and Streptococcus mutans between diabetic dialysis and non-diabetic dialysis patients and the normal population.

Patients and Methods: During November 2014 to January 2014, 85 people that referred to our medical care center entered the study. The sample included 30 diabetic dialysis, 28 nondiabetic dialysis patients and 27 healthy people. Oral saliva samples were obtained from their tongue and oral floor for microbiological examination. Patients' data were compared before and after dialysis.

Results: The amount of Lactobacillus and $S$. mutans did not show a significant difference between the three groups ( $P=0.092$ and $P=0.966$ for $S$. mutans and lactobacillus, respectively). A positive and meaningful correlation was seen between fasting blood sugar (FBS) levels and the amount of $S$. mutans in the diabetic dialysis group $(P=0.023 ; \mathrm{r}=0.413)$. A meaningful and positive correlation was also seen between the amount of blood urea nitrogen (BUN) after dialysis and the amount of oral $S$. mutans in the non-diabetic dialysis group $(P=0.03$; $\mathrm{r}=0.403)$.

Conclusion: Despite the differences in the prevalence of caries that have been reported between renal failure patients and diabetic patients, we did not find any significant difference between diabetic dialysis, non-diabetic dialysis patients and the healthy population, regarding their amount of oral cariogenic bacteria.
\end{abstract}

\section{Implication for health policy/practice/research/medical education:}

Diabetic dialysis patients tend to have a complex oral status and special attention should be given to their oral health.

Please cite this paper as: Rezazadeh F, Bazargani A, Roozbeh-Shahroodi J, Pooladi A, Arasteh P, Zamani K. Comparison of oral Lactobacillus and Streptococcus mutans between diabetic dialysis patients with non-diabetic dialysis patients and healthy people. J Renal Inj Prev. 2016;5(3):148-152. DOI: 10.15171/jrip.2016.31

\section{Introduction}

Diabetes is a common disease affecting more than 387 million people worldwide (1). The condition is associated with many complications and among them, diabetic nephropathy is one of the major complications that develops in $40 \%$ of patients with insulin dependent diabetes mellitus (DM) (2). The high prevalence of DM results in a sustained increase in the prevalence of diabetic nephropathy, which in return, causes increased kidney failure (3). Patients with DM are faced with more oral disorders than the normal population. Caries and periodontal disease are a well-documented complication of diabetes. Other complications include xerostomia, bacterial, viral and fungal infections and poor wound healing $(4,5)$. 
On the other hand, chronic renal failure is seen with several oral changes such as ammonia-like smell, dysgeusia, stomatitis, gingivitis, decreased salivary flow and parotitis (6). Although caries is not a characteristic of disease some oral lesions like lichen planus, hairy tongue and pyogenic granuloma have been reported with a higher rate in these patients (7).

The oral cavity is colonized with different species of bacteria and some of these bacteria contribute to some of the most common bacterial infections such as caries and periodontitis (8). The most important causes of dental caries are Lactobacillus and Streptococcus mutans (9).

Patients with diabetes are documented to have higher levels of dental caries due to multiple factors, on the other hand some studies have shown that patients with chronic renal failure may have lower levels of cariogenic bacteria in their oral cavity $(4,10)$.

Different results regarding the oral microflora in patients with diabetes and renal failure have been reported (1113). Ahmadieh et al (12) reported that hemodialysis and kidney transplantation could affect the oral microflora. They found that oral Streptococcus mutans and Lactobacil$l i$ were seen less frequently after kidney transplantation. In another study, the oral health of patients on hemodialysis for diabetic nephropathy and chronic glomerulonephritis was studied and they found that fewer teeth and a worse periodontal health was seen in diabetic nephropathy patients compared to other groups (4).

\section{Objectives}

Due to the discrepancy in the prevalence of dental caries among these patients, in this study we compared oral Lactobacillus and Streptococcus mutans among patients with end-stage renal disease and diabetic nephropathy, both undergoing dialysis with the normal population.

\section{Patients and Methods}

Study settings and patients

This study was conducted in Faghihi hospital, Shiraz, Iran. The study population was selected from patients that were referred to our medical care center for dialysis, during November 2014 to January 2014. Patients who received periodontal treatment during the past year, brushed their teeth or ate something within one hour prior to sampling, took antibiotics or used mouth wash in the past previous month, were seropositive or suspicious to have HIV, hepatitis B or C, chronic smokers and patients that had any other systemic disease that could have affected the oral microflora were excluded from the study. They were then divided into two groups: group 1 or the diabetic dialysis patients, group 2 or the non-diabetic dialysis patients. A third group was selected from the healthy people who were accompanying the patients during the dialysis, as the control group.

\section{Sample collection}

Oral saliva samples were collected from each patient using a swab. The swab was rotated to remove saliva from the dorsum of the tongue and the floor of the mouth. It was then immediately placed into a sterile bottle containing $1 \mathrm{~mL}$ of thioglycolate medium (HiMedia; India) in order to maintain the viability of the microorganisms collected. Within 15 minutes of collection, the swabs were transported to the microbiology laboratory for the microbiological testing.

For the enumeration of Streptococcus mutans, mitis salivarius agar (HiMedia, Indian) was used. The agar was supplemented with $0.1 \%$ potassium tellurite, bacitracin $0.2 \mathrm{U} /$ $\mathrm{mL}$, and sucrose $15 \% \mathrm{w} / \mathrm{v}$ (MSA). The samples were then diluted by 100 folds with salin, after which $10 \mu \mathrm{L}$ of the diluted samples were transferred to the colony plates. The plates were then set in microaerophilic conditions and incubated for 1 day at $37^{\circ} \mathrm{C}$.

For the Lactobacillus, Rogosa agar (HiMedia, India) was employed. For the enumeration of the bacteria, $20 \mu \mathrm{L}$ of the samples was transported to the plates. The inoculated plates were then incubated in an anaerobic jar with an anaerobic atmosphere of $85 \%$ nitrogen, $5 \%-10 \%$ carbon dioxide and $5 \%-10 \%$ oxygen for 4 days at $37^{\circ} \mathrm{C}$.

Assessment of the cultures and lab tests were all performed by a single microbiologist who was blinded to the study groups.

\section{Classification and evaluation}

The salivary levels of the $S$. mutans were classified into five groups based on the colony count: $1: 0-10^{5} \mathrm{CFU} / \mathrm{mL}$ of saliva, 2: $10^{5}-5 \times 10^{5} \mathrm{CFU} / \mathrm{mL}, 3: 5 \times 10^{5}-10^{6} \mathrm{CFU} / \mathrm{mL}, 4: 10^{6}$ $-1.5 \times 10^{6}$ and $5:>1.5 \times 10^{6}$.

The Lactobacillus were classified into six groups based on the colony count in each sample; 1 : Non-detectable, 2 : 1-10 ${ }^{3} \mathrm{CFU} / \mathrm{mL}$ saliva, $3: 10^{3}-5 \times 10^{3} \mathrm{CFU} / \mathrm{mL}, 4: 5 \times 10^{3}-10^{4}$ $\mathrm{CFU} / \mathrm{mL}$ saliva, $5: 10^{4}-1.5 \times 10^{4} \mathrm{CFU} / \mathrm{mL}$ and $6:>1.5 \times 10^{4}$. Patients' information regarding age, sex, fasting blood sugar (FBS) levels, creatinine and blood urea nitrogen (BUN) levels prior to dialysis and after dialysis (only for the case groups) were recorded and compared between the groups.

\section{Ethical issues}

The study protocol was in accordance with the declaration of Helsinki and was approved by the Ethics Committee of Shiraz University of Medical Sciences. All participants gave their informed written consent to enter the study (Ethical code\# 2232).

\section{Statistical analysis}

The statistical analysis was done using the SPSS ${ }^{\circledR}$ for windows $^{\circledR}$, version 17 (SPSS Inc., Chicago, IL, USA). The data are displayed and compared as frequency, percent, median and interquartile range. The Kruskal-Wallis test was used to compare means among the 3 groups and the chi-square test was used for comparison of means between 2 groups. The Spearman's correlation was used to assess the relationship between the frequency of each bacteria and different parameters including age, FBS, creatinine and BUN levels in the groups. A two-tailed $P$ value of less than 0.05 
was considered as statistically significant.

Result

Baseline characteristics

Eighty-five people entered the study and were evaluated. Group 1 (diabetic dialysis patients) included 30 patients, group 2 (non-diabetic dialysis patients) included 28 patients and group 3 (control group) included 27 healthy people. The patients did not display any significant difference regarding age and sex. The baseline characteristic of the participants is displayed in Table 1.

\section{Comparison of groups and correlations}

For comparison of the oral flora between the three groups, each of the two types of bacteria was classified into 5 groups as mentioned before. The results did not show a statistically significant difference in the amount of bacteria between the three groups $(P=0.092$ and $P=0.966$ for $S$. mutans and lactobacillus, respectively) (Table 1).

A statistically significant and positive correlation was seen between FBS and the amount of $S$. mutans in group 1, in other words an increase in the FBS of the diabetic dialysis patients resulted in an increase in the amount of oral S. mutans $(P=0.023 ; \mathrm{r}=0.413)$, although this correlation was not found with Lactobacillus for this group $(P=0.129$; $\mathrm{r}=0283)$.

In the non-diabetic dialysis group a meaningful and positive correlation was found between the level of BUN after dialysis and the amount of oral $S$. mutans $(P=0.03$; $\mathrm{r}=0.403)$, although this correlation was not seen between BUN before dialysis and $S$. mutans for this group of patients $(P=0.29 ; \mathrm{r}=0.205)$.
None of the other parameters showed a significant correlation with either of the bacteria $(P>0.05$; Table 2$)$.

\section{Discussion}

In our study we documented a positive correlation between the amount of FBS and the amount of oral S. mutans in the diabetic patients, furthermore the BUN after dialysis was positively correlated with the amount of oral $S$. mutans in the non-diabetic dialysis patients. No meaningful correlation between Lactobacillus and any of the disease related variables that we assessed in our study was seen.

In a recent study by Teratani et al (4) in 2013, the oral health was compared between patients with diabetic nephropathy (DN) and chronic glomerulonephritis (CGN) with a control group. They found that patients with diabetic nephropathy had overall worse oral health in compared to both CGN patients and the control group. Although both the CGN and the DN group both had a worse condition regarding salivary flow rate and total xerostomia score. The xerostomia score was worse in patients with DN compared to the CGN group. In another study in 2011 (14), comparing oral health among patients with DN and patients with other causes of chronic kidney disease (CKD) at pre-dialysis stage, they found that DN patients had higher rates of dental caries. Our study also showed a positive correlation between the amount of $S$. mutans and FBS in the diabetic patients, furthermore the rate of cariogenic bacteria did show a meaningful difference between the three groups. This could have been due to multiple reasons including that caries might not only be dependent on oral microflora and other factors such as

Table 1. Baseline characteristics and frequency of bacteria in the three groups ${ }^{a}$

\begin{tabular}{|c|c|c|c|c|}
\hline Variables & Group $1(n=30)$ & Group $2(n=28)$ & Group $3(n=27)$ & $P$ value \\
\hline Age (years) & $65 \pm 11$ & $59 \pm 14$ & $63 \pm 12$ & 0.119 \\
\hline $\operatorname{Sex}(m: f)$ & $20: 10$ & $17: 11$ & $17: 10$ & 0.893 \\
\hline FBS & $194 \pm 76$ & $94 \pm 19$ & - & $<0.001$ \\
\hline Creatinine before dialysis & $6.5 \pm 1.5$ & $7.9 \pm 2.3$ & - & 0.008 \\
\hline Creatinine after dialysis & $3.1 \pm 0.8$ & $3.5 \pm 1.1$ & - & 0.07 \\
\hline BUN before dialysis & $54 \pm 11$ & $59 \pm 17$ & - & 0.26 \\
\hline BUN after dialysis & $19 \pm 5$ & $19 \pm 5$ & - & 0.91 \\
\hline \multicolumn{4}{|c|}{ Streptococcus mutans (Frequency based on grouping ${ }^{\mathrm{b}}$ ), n (\%) } & 0.092 \\
\hline Group 1 & $2(6.7)$ & $4(14.3)$ & $1(3.7)$ & \\
\hline Group 2 & $7(23.3)$ & $10(35.7)$ & $7(25.9)$ & \\
\hline Group 3 & $7(23.3)$ & 5 (17.9) & $1(3.7)$ & \\
\hline Group 4 & $3(10)$ & $1(3.6)$ & $4(14.8)$ & \\
\hline Group 5 & $11(36.7)$ & $8(28.6)$ & $14(51.9)$ & \\
\hline \multicolumn{4}{|c|}{ Lactobacillus (Frequency based on grouping $), \mathrm{n}(\%)$} & 0.966 \\
\hline Group 1 & $17(56.7)$ & $14(50)$ & $13(48.1)$ & \\
\hline Group 2 & $4(13.3)$ & $8(28.6)$ & $5(18.5)$ & \\
\hline Group 3 & $4(13.3)$ & $2(7.1)$ & 7 (25.9) & \\
\hline Group 4 & $0(0)$ & $1(3.6)$ & $2(7.4)$ & \\
\hline Group 5 & $2(6.7)$ & $0(0)$ & $0(0)$ & \\
\hline Group 6 & $3(10)$ & $3(10.7)$ & $0(0)$ & \\
\hline
\end{tabular}

${ }^{a}$ Group 1 includes the diabetic dialysis patients; group 2 includes the non-diabetic dialysis patients; group 3 includes the control group.

${ }^{b}$ The S. mutans were divided into 5 groups based on their frequency in each group: group 1: 0-10 ; group 2 : $10^{5}-5 \times 10^{5}$; group $3: 5 \times 10^{5}-10^{6}$; group 4 : $10^{6}-1.5 \times 10^{6}$; group 5: > $1.5 \times 10^{6}$.

${ }^{\mathrm{c}}$ The Lactobacillus were divided into 6 groups based on their frequency in each group: group 1: non detectible; group 2: $1-10^{3}$; group 3: $10^{3}-5 \times 10^{3}$; group 4: $5 \times 10^{3}-10^{4}$; group 5: $10^{4}-1.5 \times 10^{4}$; group $6:>1.5 \times 10^{4}$. 
Table 2. Correlation coefficient between Streptococcus mutans and Lactobacillus frequency with differed variables in the three groups ${ }^{a}$

\begin{tabular}{|c|c|c|c|c|c|c|c|c|c|c|c|c|}
\hline \multirow{2}{*}{ Variables } & \multicolumn{4}{|c|}{ Group $1(n=30)$} & \multicolumn{4}{|c|}{ Group $2(n=28)$} & \multicolumn{4}{|c|}{ Group 3 ( $n=27)$} \\
\hline & SM (r) & $P$ value & LB (r) & $P$ value & SM.(r) & $P$ value & LB (r) & $P$ value & SM. (r) & $P$ value & LB (r) & $P$ value \\
\hline FBS & 0.413 & 0.023 & 0.283 & 0.12 & 0.151 & 0.44 & -0.234 & 0.23 & - & - & - & - \\
\hline Creatinine before dialysis & 0.16 & 0.398 & 0.098 & 0.60 & -0.66 & 0.74 & -0.053 & 0.79 & - & - & - & - \\
\hline Creatinine after dialysis & -0.088 & 0.643 & 0.151 & 0.42 & -0.64 & 0.74 & 0.152 & 0.44 & - & - & - & - \\
\hline BUN before dialysis & -0.025 & 0.894 & 0.22 & 0.24 & 0.205 & 0.29 & -0.095 & 0.63 & - & - & - & - \\
\hline BUN after dialysis & -0.173 & 0.361 & -0.25 & 0.17 & 0.403 & 0.03 & 0 & 0.99 & - & - & - & - \\
\hline Age & 0.041 & 0.828 & -0.258 & 0.168 & 0.396 & 0.037 & -0.041 & 0.637 & -0.054 & 0.78 & -0.422 & 0.029 \\
\hline
\end{tabular}

Abbreviations: FBS, fasting blood sugar; BUN, blood urea nitrogen; SM, Streptococcus mutans; LB, Lactobacillus.

${ }^{a}$ Group 1 includes the diabetic dialysis patients; group 2 includes the non-diabetic dialysis patients; group 3 includes the control group.

salivary flow rates and the amount of sugar in the saliva could have affected the results, furthermore patients with renal failure tend to have higher $\mathrm{pH}$ of the oral cavity due to their uremic state which could have provided protection against dental caries (10).

In one study in 2010 (12), a higher rate of S. mutans was seen in the control group compared to patients with hemodialysis and renal transplant and a lower rate of lactobacilli was seen in the control group, although these differences were not significantly meaningful. This result was similar to our findings. This was further supported by other studies that found a lower rate of dental caries in end stage renal failure and renal transplant patients $(15,16)$. In one review by Soell et al in 2007 (17), they concluded that diabetic patients have an overall worse oral health in wise of dental caries when compared to healthy individuals. They concluded that this is due to two factors of increased oral sugar levels and increased S. mutans and by controlling these two factors oral caries becomes the same as in healthy individuals.

Similar to the previous study, one study in 2013 (18), evaluated the dental caries of diabetic (controlled and uncontrolled) and non-diabetic patients. They found that the diabetic group had the highest amount of oral S. mutans and lactobacilli in comparison to the other groups $(P<0.001)$. They also found that the amount of caries in the uncontrolled diabetic group correlated positively with the amount of HbAlc $(\mathrm{r}=0.574$ and $P=0.003)$, which is consistent with our findings regarding the correlations between the amount of $S$. mutans and FBS in our diabetic nephropathy group $(\mathrm{r}=0.413$ and $P=0.023)$. Diabetic patients are documented to be at higher risks of developing conditions like periodontitis and xerostomia apart from the added nephropathy which acts as a separate risk factor for poor hygiene $(19,20)$. As shown in the previously mentioned studies patients with diabetes have lower salivary flow rates, which put them at higher risks of caries. Similar result was also seen in other studies evaluating the cariogenic bacteria in diabetic patients (21).

The findings in our study suggest that the BUN after dialysis may reflect the oral health status, so further studies should be conducted to evaluate if BUN after dialysis could be used as an indicator of caries for non-diabetic dialysis patients. We also found that the higher blood sugar in diabetic patients reflects a worse oral health and this shows that the less the diabetes is controlled the more oral caries is expected in this group of patients. We found that the non-diabetic patients had a lower amount of cariogenic bacteria than the control group. In the diabetic dialysis patients, considering their diabetes, we expected a worse oral health as indicated by some of the previously mentioned studies $(4,12,17)$, on the other hand considering their renal failure we expected less oral caries. In our study we found no difference in the amount of cariogenic bacteria in these patients compared to the other groups showing that these patients are complicated regarding their oral health status and special attention should be given to these patients' dental health.

Larger scale studies considering different types of renal failure, patients with concomitant diabetes and in patients with different socio-economic status should be conducted to further compare the difference in the oral health of these patients.

\section{Conclusion}

Despite the differences in the prevalence of caries that have been reported between renal failure patients and diabetic patients, we did not find any significant difference between diabetic dialysis and non-diabetic dialysis patients, regarding their amount of oral cariogenic bacteria. In renal failure patients the BUN level after dialysis was positively correlated with their amount of $S$. mutans, furthermore diabetic patients displayed more S. mutans by an increase in their FBS.

\section{Limitations of the study}

Our study had some limitations. First the types of medications that the patients were using were not taken into consideration. This may have had adverse effects on their oral health and might have affected the results of our study. Another factor that might have affected the oral caries was the socio-economic status as indicated by one study (22). It was not taken into consideration as a factor in our study, although most of our recruited patients had a poor socioeconomic status.

\section{Acknowledgments}

The authors would like to thank the vice-chancellor of Shiraz University of Medical Sciences for their financial support of the study (grant\# 7027). This study is based on the 
thesis for the D.M.D of Dr. Ali Pooladi. The authors would also like to thank Dr. Mehrdad Vosoogh from the Dental Research Development Center of the School of Dentistry for his contribution in the statistical analysis. The authors also like to thank Dr. Shahram Hamedani (DDS, MSC) for his editorial assistance in the manuscript.

\section{Authors' contribution}

All authors contributed to design of the research. AL, AB and $\mathrm{KZ}$ conducted the research. PA drafted the article. JR aided in the scientific revision of the manuscript. All authors read, revised and approved the final manuscript.

\section{Conflicts of interest}

The authors declared no competing interests.

\section{Ethical considerations}

Ethical issues (including plagiarism, data fabrication, double publication) have been completely observed by authors.

\section{Funding/Support}

This is a thesis related to Shiraz University of Medical Sciences, School of Dentistry. (Institutional Review Board code \#7027).

\section{References}

1. International Diabetes Federation. IDF Diabetes Atlas. Belgium: International Diabetes Federation; 2014.

2. Byun JM, Lee CH, Lee SR, Moon JY, Lee SH, Lee TW, et al. Renal outcomes and clinical course of nondiabetic renal diseases in patients with type 2 diabetes. Korean J Intern Med. 2013;28:565-72. doi: 10.3904/kjim.2013.28.5.565.

3. Afkarian M, Sachs MC, Kestenbaum B, Hirsch IB, Tuttle KR, Himmelfarb J, et al. Kidney disease and increased mortality risk in type 2 diabetes. J Am Soc Nephrol. 2013;24:302-8. doi: 10.1681/asn.2012070718.

4. Teratani G, Awano S, Soh I, Yoshida A, Kinoshita N, Hamasaki T, et al. Oral health in patients on haemodialysis for diabetic nephropathy and chronic glomerulonephritis. Clin Oral Investig. 2013;17:483-9. doi: 10.1007/s00784012-0741-1.

5. Duran I, Erdemir EO. Periodontal treatment needs of patients with renal disease receiving haemodialysis. Int Dent J. 2004;54:274-8. doi: 10.1111/j.1875-595X.2004. tb00293.x.

6. Al-Nowaiser A, Roberts GJ, Trompeter RS, Wilson M, Lucas VS. Oral health in children with chronic renal failure. Pediatr Nephrol. 2003;18:39-45 doi: 10.1007/s00467-0020999-7.

7. Little J. Dental management of the medically compromised patient. 7th ed. St. Louis: Mosby/Elsevier; 2008. p. 628.

8. Sahebjamee M, Shakur Shahabi M, Nikoobakht MR, Momen Beitollahi J, Mansourian A. Oral lesions in kidney transplant patients. Iran J Kidney Dis. 2010;4:232-6.

9. Tanner AC, Mathney JM, Kent RL, Chalmers NI, Hughes
CV, Loo CY, et al. Cultivable anaerobic microbiota of severe early childhood caries. Journal of clinical microbiology. 2011;49:1464-74. doi: 10.1128/jcm.02427-10.

10. Castillo A, Mesa F, Liebana J, Garcia-Martinez O, Ruiz S, Garcia-Valdecasas J, et al. Periodontal and oral microbiological status of an adult population undergoing haemodialysis: a cross-sectional study. Oral Dis. 2007;13:198-205 doi: 10.1111/j.1601-0825.2006.01267.x.

11. Craig RG, Kotanko P, Kamer AR, Levin NW. Periodontal diseases--a modifiable source of systemic inflammation for the end-stage renal disease patient on haemodialysis therapy? Nephrol Dial Transplant. 2007;22:312-5.

12. Ahmadieh A, Baharvand M, Fallah F, Djaladat H, Eslani M. Oral microflora in patients on hemodialysis and kidney transplant recipients. Iran J Kidney Dis. 2010;4:227-31.

13. Bayraktar G, Kurtulus I, Kazancioglu R, Bayramgurler I, Cintan S, Bural C, et al. Evaluation of periodontal parameters in patients undergoing peritoneal dialysis or hemodialysis. Oral Dis. 2008;14:185-9. doi: 10.1111/j.16010825.2007.01372.x.

14. Vesterinen M, Ruokonen H, Furuholm J, Honkanen E, Meurman JH. Oral health in predialysis patients with emphasis on diabetic nephropathy. Clin Oral Investig.. 2011;15:99-104. doi: 10.1007/s00784-009-0360-7.

15. Lucas VS, Roberts GJ. Oro-dental health in children with chronic renal failure and after renal transplantation: a clinical review. Pediatr Nephrol. 2005;20:1388-94 doi: 10.1007/s00467-005-1929-2.

16. Al Nowaiser A, Lucas VS, Wilson M, Roberts GJ, Trompeter RS. Oral health and caries related microflora in children during the first three months following renal transplantation. Int J Paediatr Dent. 2004;14:118-26. doi: 10.1111/j.1365-263X.2004.00534.x.

17. Soell M, Hassan M, Miliauskaite A, Haikel Y, Selimovic D. The oral cavity of elderly patients in diabetes. Diabetes Metab. 2007;33:S10-8.

18. Al-Khayoun JD, Diab BS. Dental caries, Mutans Streptococci, Lactobacilli and salivary status of type1 diabetic mellitus patients aged 18-22 years in relation to glycated haemoglobin. Journal of Baghdad College of Dentistry. 2013;25:153-8.

19. Tanwir F, Altamash M, Gustafsson A. Effect of diabetes on periodontal status of a population with poor oral health. Acta Odontol Scand. 2009;67:129-33. doi: 10.1080/00016350802208406.

20. Kaur G, Holtfreter B, Rathmann W, Schwahn C, Wallaschofski H, Schipf S, et al. Association between type 1 and type 2 diabetes with periodontal disease and tooth loss. J Clin Periodontol. 2009;36:765-74 doi: 10.1111/j.1600051X.2009.01445.x.

21. Kampoo K, Teanpaisan R, Ledder RG, McBain AJ. Oral bacterial communities in individuals with type 2 diabetes who live in southern Thailand. Appl Environ Microbiol. 2014;80:662-71 doi: 10.1128/aem.02821-13.

22. Zukanović A, Muratbegović A, Kobaslija S, Marković N, Ganibegović M, Beslagić E. Relationships between socioeconomic backgrounds, caries associated microflora and caries experience in 12-year-olds in Bosnia and Herzegovina in 2004. Eur J Paediatr Dent. 2008;9:118-24.

Copyright (C) 2016 The Author(s); Published by Nickan Research Institute. This is an open-access article distributed under the terms of the Creative Commons Attribution License (http://creativecommons.org/licenses/by/4.0), which permits unrestricted use, distribution, and reproduction in any medium, provided the original work is properly cited. 